\title{
The Correlation of Tunnel Position, Orientation and Tunnel Enlargement in Outside-in Single-Bundle Anterior Cruciate Ligament Reconstruction
}

\author{
Young Won Ko, MD, Seung Jun Rhee, MD, In Woo Kim, MD, and Jae-Doo Yoo, MD \\ Department of Orthopedic Surgery, Ewha Womans University Mokdong Hospital, Seoul, Korea
}

\begin{abstract}
Purpose: Tunnel widening after anterior cruciate ligament (ACL) reconstruction is a frequently described phenomenon. The possible etiology is multi-factorial with some mechanical and biological factors. Among those, we intended to determine the relation between the location and orientation of the femoral tunnel and the femoral tunnel enlargement after outside-in single-bundle ACL reconstruction.

Materials and Methods: A retrospective study including 42 patients who received single-bundle ACL reconstruction with the outside-in technique was conducted. Femoral and tibial tunnel locations were evaluated with the quadrant method and bird's-eye view using volume-rendering computed tomography. The angle and diameter of bone tunnel and the degree of tunnel enlargement were evaluated using standard radiographs.

Results: The degree of femoral tunnel enlargements were $42 \%$ and $36 \%$ on the anteroposterior (AP) and lateral radiographs, respectively, and the degree of tibial tunnel enlargements were $22 \%$ and $23 \%$, respectively. Shallower location of the femoral tunnel was significantly correlated with greater femoral tunnel enlargement on the AP radiograph $(\mathrm{r}=0.998, \mathrm{p}=0.004)$ and the lateral radiograph $(\mathrm{r}=0.72, \mathrm{p}=0.005)$ as was the higher location of the femoral tunnel on the AP radiograph $(r=-0.47, p=0.01)$ and the lateral radiograph $(r=-0.36, p=0.009)$ at 12 months after surgery.

Conclusions: This study revealed that more anterior and higher location and more horizontal orientation of the femoral tunnel in coronal plane could result in widening of the femoral tunnel in outside-in single-bundle ACL reconstruction.
\end{abstract}

Keywords: Knee, Anterior cruciate ligament, Outside-in technique, Tunnel enlargement

\section{Introduction}

Bone tunnel widening after single-bundle anterior cruciate ligament (ACL) reconstruction is known as a frequently described phenomenon regardless of reconstructed graft materials ${ }^{1)}$. The etiology of tunnel widening is multi-factorial with several mechanical and biological contributing factors ${ }^{2)}$. It is also unclear whether there is a correlation between tunnel widening and tun-

Received May 20, 2015; Revised (1st) September 26, 2015;

(2nd) October 4, 2015; Accepted October 5, 2015

Correspondence to: Jae-Doo Yoo, MD

Department of Orthopedic Surgery, Ewha Womans University Mokdong Hospital, 1071 Anyangcheon-ro, Yangcheon-gu, Seoul 07985, Korea

Tel: +82-2-2650-6142, Fax: +82-2-2642-0349

E-mail: koreanknee@gmail.com

This is an Open Access article distributed under the terms of the Creative Commons Attribution Non-Commercial License (http://creativecommons.org/licenses/by-nc/4.0/) which permits unrestricted non-commercial use, distribution, and reproduction in any medium, provided the original work is properly cited. nel aperture location or tunnel obliquity. Accurate placement of the femoral tunnel is an integral part of ACL reconstruction and efforts are made to replicate the anatomy of the native ACL to achieve superior clinical outcome after reconstruction ${ }^{3)}$. The traditional transtibial technique has some advantages of enabling to simplify the procedure and reducing surgical time and surgical trauma ${ }^{4}$. However, this technique may fail to replicate native ligament origins and result in more vertically oriented femoral tunnels because femoral tunnel creation is determined by tibial tunnel creation due to coupled drilling ${ }^{5}$. The outside-in 2-incision tibial tunnel-independent technique has been developed to overcome the limitations of transtibial technique and improve the accuracy of tunnel placement. In recent studies, successful results and more anatomical location of the femoral tunnel after reconstruction using the outside-in technique have been reported ${ }^{6,7}$. The purpose of this study was to evaluate the correlation between tunnel widening and tunnel aperture location and tunnel obliquity. We hypothesized that femoral tunnel position and orientation would significantly affect femoral tunnel enlargement. 


\section{Materials and Methods}

\section{Patient Selection and Demographics}

From January 2010 to December 2012, 86 patients with unilateral ACL deficiency who underwent outside-in single-bundle ACL reconstruction using 4-strand hamstring (semitendinosus and gracilis) tendon autograft (13 patients) and fresh frozen tibialis tendon allograft (73 patients) performed by a senior surgeon (Yoo) were selected. Consecutive patients in whom examination of postoperative computed tomography (CT) scans was possible were enrolled in this study without randomization. The exclusion criteria included: 1) multiple ligament injury requiring concomitant surgery; 2) evidence of osteoarthritis on radiographs; 3 ) revision ACL reconstruction; 4) tunnel entrance that could not be clearly identified in the three-dimensional (3D) CT model; 5) patients without follow-up radiograph; 6) a tunnel that could not be discernible on radiographs; and 7) incorrectly positioned anteroposterior (AP) and lateral radiographs. Finally, 44 patients were excluded from the initial 86 patients; therefore, 42 patients with 4 autografts and 38 allografts were included in this study.

\section{Surgical Technique}

After careful diagnostic arthroscopy, the tibial tunnel was prepared as in the traditional transtibial technique. For the femoral tunnel, the femoral footprint was identified by minimal debride-

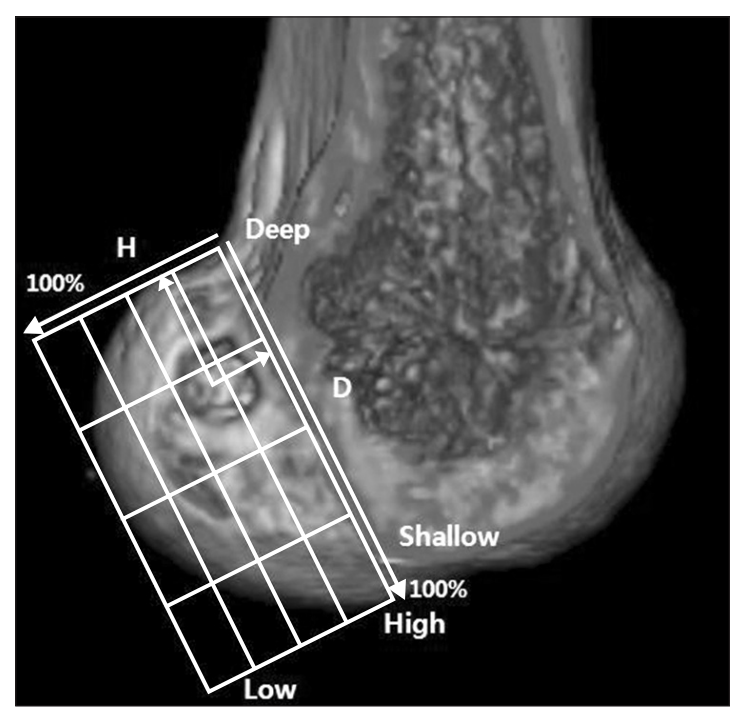

Fig. 1. The location of the center of the femoral tunnel was defined as the percentage of the distance from the most posterior contour in reference to the total length of the lateral condyle parallel to the Blumensaat's line and the percentage of the distance from the intercondylar roof with respect to the total depth of the intercondylar notch perpendicular to the Blumensaat's line. H: perpendicular to the Blumensaat's line, D: parallel to the Blumensaat's line. ment of the ACL stump or bony landmarks including the lateral intercondylar ridge and lateral bifurcate ridge. The femoral socket was made with the outside-in technique using retractable retrograde cutting bits (FlipCutter; Arthrex, Naples, FL, USA) that require only a portal-sized stab wound. After positioning of the grafts, TightRope (Arthrex) was used for femoral fixation of the graft and double tibial fixation was performed using a bioabsorbable interference screw and a spiked washer and screw with the knee in $30^{\circ}$ of flexion position.

\section{Tunnel Position on 3D CT}

CT scanning was performed with the knee in extended position within 1 week after surgery. Images were taken by a SOMATOM Sensation Caradiac 64 (Siemens, Munich, Germany) and conventional volume-rendering $3 \mathrm{D}$ surface models were obtained using 3D-inspace software (Siemens). The files were converted to Digital Imaging and Communication in Medicine format and transmitted to picture archiving and communication system (PACS; PiViewSTAR 5.0, INFINITT, Seoul, Korea) for geometric measurements. From these 3D CT images, the placements of femoral and tibial tunnels were assessed by determining the centers of the femoral and tibial tunnel apertures. To evaluate the femoral tunnel placement, we used the quadrant method described by Bernard et al. ${ }^{8}$ on a true medial view of the femur (Fig. 1). To evaluate the tibial tunnel placement, we used a true proximal-todistal view on the tibial plateau as described by Tsuda et al. ${ }^{9}$ (Fig. 2).

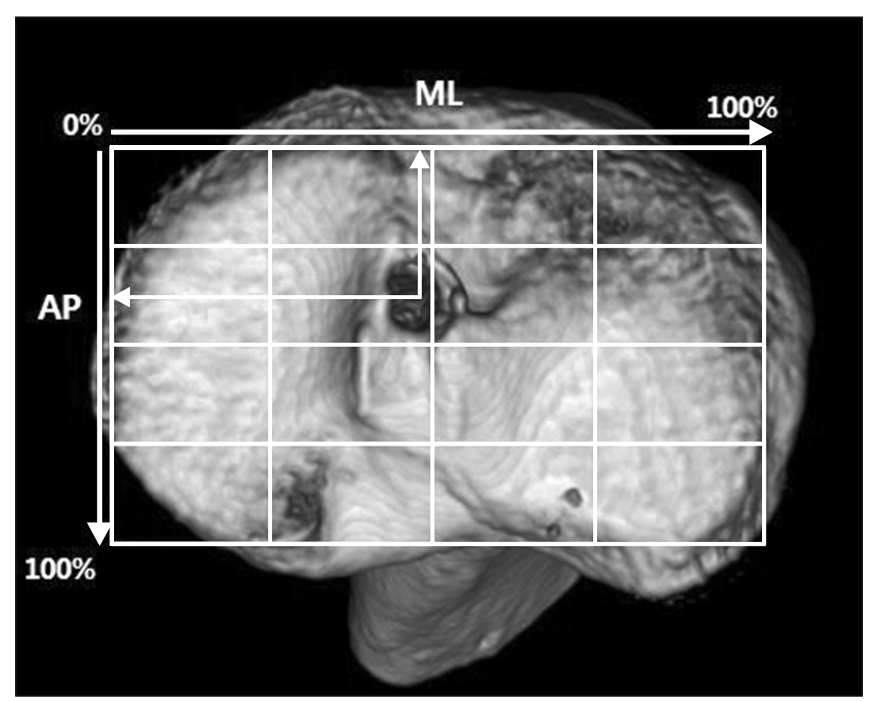

Fig. 2. The location of the center of the tibial tunnel was defined as the percentage of the distance from the most medial contour with respect to the mediolateral (ML) width of the tibial plateau and the percentage of the distance from the most anterior contour with respect to the anteroposterior (AP) length of the tibial plateau. 
To improve measurement accuracy, repeated measurements were performed.

\section{Evaluation of Tunnel Angles}

The standard AP and lateral radiographs taken at 3 month after surgery were used for evaluation of the tunnel angles. If the radiographic images were neither available nor appropriate for measurement, the radiographs obtained at 6 weeks or 6 months after surgery were used. First, the tunnel axis defined as a line passing each central point of two outlets of the tunnel was determined to measure the tunnel angle on AP and lateral radiographs. Then, the tunnel angle defined as the angle between the tunnel axis and the joint surface or femoral shaft axis was assessed (Fig. 3).

\section{Evaluation of Tunnel Widening}

The bone tunnel diameters were measured to evaluate widening of the femoral and tibial tunnels on standard AP and lateral radiographs taken immediately postoperatively and at 3 months, 6 month, and 12 months after surgery. Measurements were performed with a digital radiography system (PACS), and a built-in digital ruler was used to measure the true size on the computer screen (Fig. 4). If the bone tunnel was not identified clearly on radiographs obtained immediately postoperatively, the tunnel diameter was substituted by the final size of the dilator used in the operation. The percentage change in tunnel diameter between radiographs taken immediately postoperatively and at 12 months after surgery was defined as the degree of tunnel enlargement.
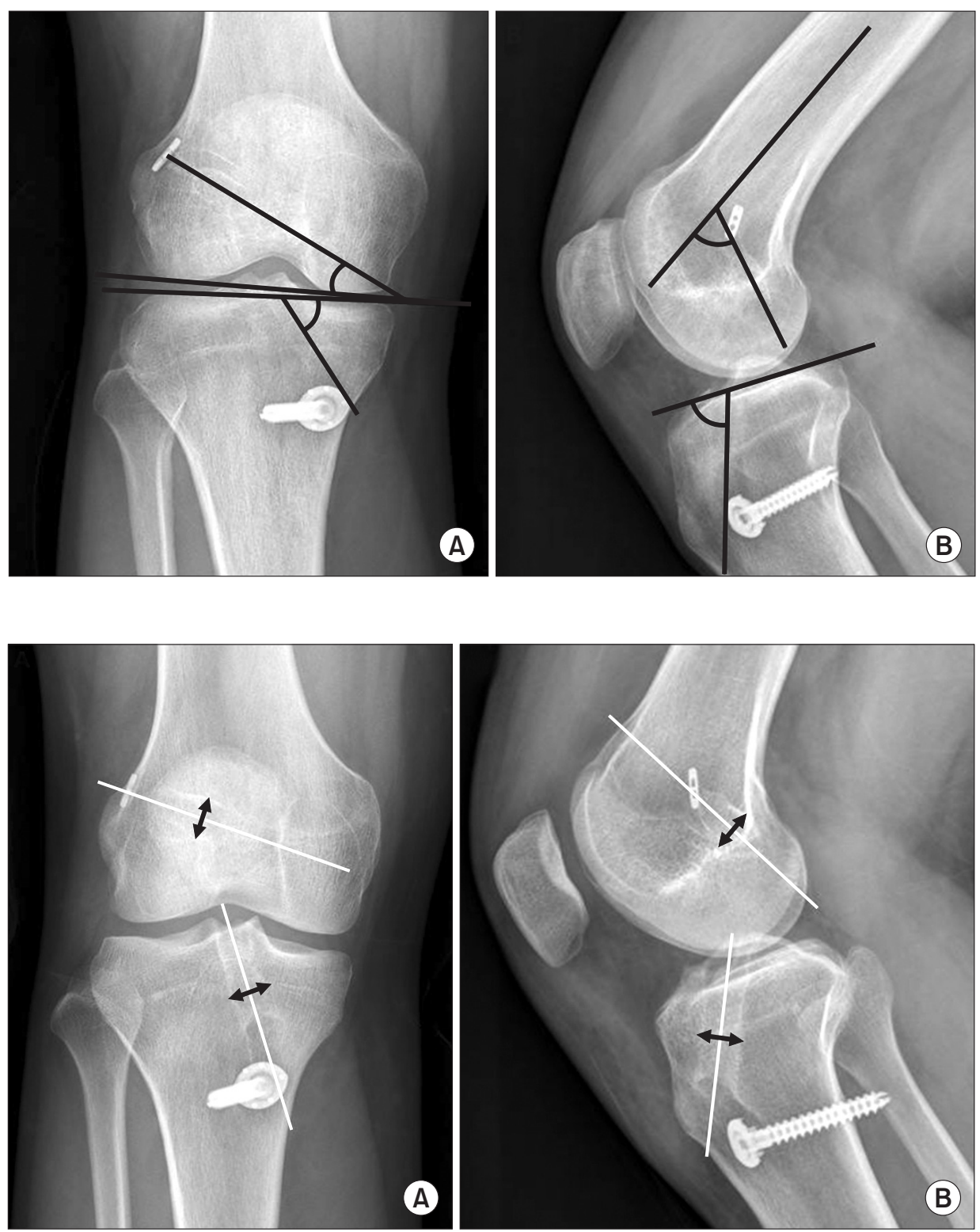

Fig. 3. (A) The femoral and tibial tunnel angles on the anteroposterior radiographs were defined as the angles between the tunnel axis and each joint surface. (B) The femoral tunnel angle on the lateral radiograph was defined as the angle between the femoral tunnel axis and the femoral shaft axis. The tibial tunnel angle on the lateral radiograph was defined as the angle between the tibial tunnel axis and the tibial joint surface.
Fig. 4. The bone tunnel diameters on the anteroposterior radiograph (A) and the lateral radiograph (B) were defined as the distances between the two sclerotic bony margins at the widest diameter perpendicular to the tunnel axis. 


\section{Statistical Analysis}

The SPSS ver. 18.0 (SPSS Inc., Chicago, IL, USA) was used. Descriptive statistics were performed for continuous variables including age, length of follow-up, tunnel position, tunnel angle, and tunnel enlargement. If the parameters were normally distributed, the Pearson correlations were used to determine the relations between tunnel enlargement, tunnel position, tunnel angle, and clinical outcomes. Spearman rank correlation coefficients were used to evaluate the relations if the parameters were not normally distributed. Test-retest reliability was assessed using Pearson correlations.

\section{Results}

\section{Patient Demographics}

There were total 42 patients enrolled in the study (33 males and 9 females) with a mean age of 33 years (range, 15 to 56 years). Their mean height and weight ( \pm standard deviation) were $169 \pm 8$ $\mathrm{cm}$ and $68 \pm 7 \mathrm{~kg}$, respectively.

\section{Tunnel Position}

The center of the femoral tunnel was located at $32.06 \% \pm 7.78 \%$ in the shallow/deep direction along Blumensaat's line and at $34.35 \% \pm 8.78 \%$ in the high/low direction from the intercondylar roof. The center of the tibial tunnel was located at $38.97 \% \pm 8.83 \%$ of the distance from the anterior edge of the tibia in the AP direction and at $48.48 \% \pm 3.25 \%$ in the mediolateral direction. The intra-class correlation coefficient of test-retest reliability was 0.91 and considered acceptable.

\section{Tunnel Angle}

The mean femoral tunnel angle was $25.57^{\circ} \pm 14.48^{\circ}$ on the AP radiograph and $65.31^{\circ} \pm 14.44^{\circ}$ on the lateral radiograph. The mean tibial tunnel angle was $59.16^{\circ} \pm 7.0^{\circ}$ on the AP radiograph and $59.05^{\circ} \pm 8.62^{\circ}$ on the lateral radiograph.

\section{Tunnel Enlargement}

The mean femoral tunnel diameter was $9.09 \pm 0.5 \mathrm{~mm}$ on the AP radiograph and $9.08 \pm 0.4 \mathrm{~mm}$ on the lateral radiograph immediately postoperatively and $12.91 \pm 2.3 \mathrm{~mm}$ on the AP radiograph and $12.33 \pm 2.0 \mathrm{~mm}$ on the lateral radiograph at 12 months after surgery. The mean tibial tunnel diameter was $9.18 \pm 0.6 \mathrm{~mm}$ on the AP radiograph and $9.18 \pm 0.5 \mathrm{~mm}$ on the lateral radiograph immediately postoperatively and $11.24 \pm 1.6 \mathrm{~mm}$ on the AP radiograph and $11.30 \pm 1.5 \mathrm{~mm}$ on the lateral radiograph at 12 months after surgery. The degree of femoral tunnel enlargement on the
AP radiograph and lateral radiograph was $42 \%$ and $36 \%$, respectively, and the degree of tibial tunnel enlargement was $22 \%$ and $23 \%$, respectively. The femoral and tibial tunnel enlargements were significantly correlated with each other on the AP $(r=0.548$, $\mathrm{p}=0.003)$ and lateral radiographs $(\mathrm{r}=0.480, \mathrm{p}=0.01)$. The degree of tunnel enlargement was significantly greater in the femur than in the tibia $(\mathrm{p}<0.05)$. Serial changes in femoral and tibial tunnel enlargement according to timeline showed that most of the enlargement of the bone tunnel occurred during the first 6 months after surgery and the increase in tunnel enlargement decreased afterwards until 12 months after surgery.

\section{Correlation between Tunnel Placement and Tunnel Enlargement}

Shallower position of the femoral tunnel was significantly correlated with femoral tunnel enlargement on the AP radiograph $(\mathrm{r}=0.998, \mathrm{p}=0.004)$ and the lateral radiograph $(\mathrm{r}=0.72, \mathrm{p}=0.005)$ at 12 months after surgery. Higher position of the femoral tunnel was significantly correlated with femoral tunnel enlargement on the AP radiograph $(\mathrm{r}=-0.47, \mathrm{p}=0.01)$ and the lateral radiograph $(\mathrm{r}=-0.36, \mathrm{p}=0.025)$ at 12 months after surgery.

\section{Correlation between Tunnel Angle and Tunnel Enlargement}

Femoral tunnel angles on AP radiographs were significantly correlated with femoral tunnel enlargement assessed on the AP radiographs $(\mathrm{r}=-0.86, \mathrm{p}=0.003)$ and lateral radiographs $(\mathrm{r}=-0.73$, $\mathrm{p}=0.018$ ) at 12 months after surgery. The femoral tunnel angle on the lateral radiograph and the tibial tunnel angle were not statistically related to tunnel enlargement.

\section{Discussion}

In this study, the degree of femoral tunnel enlargement during 12 months after surgery was $42 \%$ on the AP radiograph and $36 \%$ on the lateral radiograph, and the degree of tibial tunnel enlargement was $22 \%$ and $23 \%$, respectively. We compared the tunnel diameter assessed on the radiographs obtained immediately postoperatively with that taken at 12 months after surgery to evaluate the degree of tunnel enlargement because tunnel widening had been known to occur mostly during the first 3 to 6 months, progress until 1 year after surgery, and then remain steady afterwards ${ }^{2)}$. In our study, the serial changes of femoral and tibial tunnel enlargement according to timeline also showed that most of the enlargement of the bone tunnel occurred during the first 6 months after surgery and the rate of tunnel enlargement decreased afterwards until 12 months after surgery. In agreement 
with previous studies ${ }^{10)}$, our results also demonstrated that tunnel enlargement was more evident in the femoral side than in the tibial side. The cause of this phenomenon can be explained by the concept of force redirection and synovial fluid propagation. First, the graft at the intraarticular outlet of the femoral tunnel was more bent than that of the tibial tunnel, which applied greater compressive force on the edge of the tunnel ${ }^{11}$. Second, if preserved, remnant tissue at the tibial insertion, which is thicker than that at the femoral insertion, may limit synovial fluid propagation within the tibial tunnel. These two biomechanical and biological factors can contribute to greater enlargement of the femoral tunnel.

The anatomical placement of bone tunnel is a major concern in ACL reconstruction. The quadrant method described by Bernard et $\mathrm{al}^{8}{ }^{8}$ is the most widely used method for measuring femoral tunnel position. Colombet et al. ${ }^{12)}$ reported that the anteromedial bundle was located at $26.4 \%$ in the shallow/deep direction and at $25.3 \%$ in the high/low direction and the posterolateral bundle was located at $32.3 \%$ of the length and at $47.6 \%$ of the height in their cadaveric study. Zantop et al. ${ }^{13)}$ and Forsythe et al. ${ }^{3)}$ also reported similar results in their cadaveric studies. In our study, the center of the femoral tunnel was placed at $32.06 \%$ of the length and at $34.35 \%$ of the height. These results were within the range of those of the above studies. For evaluation of tibial tunnel placement, the method suggested by Forsythe et al. ${ }^{3)}$ was used. In our study, the center of tibial tunnel was placed at $38.97 \%$ of the distance in the AP direction and at $48.48 \%$ in the mediolateral direction. Tsukada et al. ${ }^{7}$ reported that the anteromedial bundle was located at $37.6 \%$ in the AP direction and at $46.5 \%$ in the mediolateral direction and the posterolateral bundle was located at $50.1 \%$ and $51.2 \%$, respectively in their cadaveric study on anatomic tibial footprint of the ACL. Our results were within the range of the results of their study (Table 1).

There are some literatures focused on evaluation of the femoral tunnel placement in ACL reconstruction using either the transtibial technique or the anteromedial portal technique ${ }^{4,5,14-18)}$. Compared to the data documented in these studies, our results of femoral tunnel placement in ACL reconstruction using the outside-in technique are deeper and lower than those in ACL using the transtibial technique. At the same time, our results were similar to those of ACL using the anteromedial portal technique with the exception of the study of $\mathrm{Xu}$ et al. ${ }^{18)}$, which showed higher placement of the femoral tunnel (Table 2). This is consistent with the study of Chang el al. ${ }^{19)}$, which demonstrated that, compared with the anteromedial portal technique, the outside-in technique can afford a similar femoral tunnel position with a reduced femoral tunnel length. Anatomic femoral tunnel placement is more difficult to achieve with the transtibial technique because of the constraint by the tibial tunnel. The transtibial technique tends to result in higher and shallower positions of the femoral tunnel, which then causes greater laxity of the graft and enlargement of the femoral tunnel ${ }^{5,7,12,13)}$. Similarly, in our study, the outside-in technique also caused greater tunnel widening when the femoral tunnel position was shallower and higher. This is why positioning of the femoral tunnel through the anteromedial portal technique or the outside-in technique have been preferred by some authors despite the popularity and simplicity of the transtibial technique. However, femoral tunnels created through the anteromedial portal are more horizontal in the coronal plane and more vertical in the sagittal plane due to more horizontal drilling in the flexed position of the knee ${ }^{20)}$. It has been also known that with the anteromedial portal technique, the vertically oriented femoral tunnels in sagittal plane may lead to a breach of the posterior wall of the lateral condyle and result in a short tunnel ${ }^{14)}$. In addition, reaming debris and poor fluid flow cause poor arthroscopic visualization in hyperflexion, which can increase the risk of iatrogenic damage to the medial femoral condylar cartilage ${ }^{20)}$. The outsidein tibial tunnel-independent technique has been theorized to

Table 1. Comparison of Anterior Cruciate Ligament Footprint in Cadaveric Studies

\begin{tabular}{|c|c|c|c|c|c|c|c|c|}
\hline \multirow{3}{*}{ Study } & \multicolumn{4}{|c|}{ Center of femoral footprint (\%) } & \multicolumn{4}{|c|}{ Center of tibial footprint (\%) } \\
\hline & \multicolumn{2}{|c|}{$\begin{array}{c}\text { Parallel to } \\
\text { Blumensaat's line (D) }\end{array}$} & \multicolumn{2}{|c|}{$\begin{array}{c}\text { Perpendiculat to } \\
\text { Blumensaat's line }(\mathrm{H})\end{array}$} & \multicolumn{2}{|c|}{ Anterior to posterior } & \multicolumn{2}{|c|}{ Medial to lateral } \\
\hline & $\mathrm{AM}$ & PL & $\mathrm{AM}$ & PL & $\mathrm{AM}$ & PL & $\mathrm{AM}$ & PL \\
\hline Forsythe et al. ${ }^{3)}$ & 21.7 & 35.1 & 33.2 & 55.3 & 25.0 & 46.4 & 50.5 & 52.4 \\
\hline Tsukada et al. $^{7)}$ & & & & & 37.6 & 50.1 & 46.5 & 51.2 \\
\hline Colombet et al. $^{12)}$ & 26.4 & 32.3 & 25.3 & 47.6 & & & & \\
\hline Zantop et al. ${ }^{13)}$ & 18.5 & 29.3 & 22.3 & 53.6 & & & & \\
\hline Our study & \multicolumn{2}{|c|}{32.06} & \multicolumn{2}{|c|}{34.35} & \multicolumn{2}{|c|}{38.97} & \multicolumn{2}{|c|}{48.48} \\
\hline
\end{tabular}

D: femur 'D' line, $\mathrm{H}$ : femur 'H' line, AM: anteromedial, PL: posterolateral. 
Table 2. Comparison of Femoral Tunnel Positions in Previous Studies

\begin{tabular}{|c|c|c|c|c|c|c|c|}
\hline \multirow{2}{*}{ Study } & \multirow{2}{*}{$\begin{array}{c}\text { Femoral tunnel } \\
\text { technique }\end{array}$} & \multicolumn{3}{|c|}{ Parallel to Blumensaat's line (D) } & \multicolumn{3}{|c|}{ Perpendiculat to Blumensaat's line $(\mathrm{H})$} \\
\hline & & $\mathrm{AM}$ & PL & Center & $\mathrm{AM}$ & PL & Center \\
\hline \multirow[t]{2}{*}{ Ahn et al. ${ }^{4)}$} & $\mathrm{TT}$ & & & 36.49 & & & 7.71 \\
\hline & OI & & & 24.71 & & & 27.08 \\
\hline Kopf et al. ${ }^{5)}$ & TT & & & 37.20 & & & 11.30 \\
\hline \multirow[t]{2}{*}{ Takeda et al. $^{14)}$} & $\mathrm{TT}$ & 24.90 & 34.00 & 29.45 & 12.00 & 42.50 & 27.25 \\
\hline & $\mathrm{AM}$ & 20.90 & 29.50 & 25.20 & 18.10 & 47.70 & 32.90 \\
\hline Kawaguchi et al. $^{15)}$ & OI & 27.90 & 37.70 & 32.80 & 19.40 & 53.00 & 36.20 \\
\hline Lee et al. ${ }^{16)}$ & TT & & & 32.94 & & & 41.89 \\
\hline Youm et al. ${ }^{17)}$ & TT & & & 29.60 & & & 37.90 \\
\hline \multirow[t]{2}{*}{$\mathrm{Xu}$ et al. ${ }^{18)}$} & $\mathrm{TT}$ & & & 33.50 & & & 17.30 \\
\hline & $\mathrm{AM}$ & & & 27.50 & & & 23.30 \\
\hline Our study & OI & & & 32.60 & & & 34.35 \\
\hline
\end{tabular}

Values are presented as percentage.

D: femur ' $D$ ' line, $\mathrm{H}$ : femur ' $\mathrm{H}$ ' line, AM: anteromedial, PL: posterolateral, TT: transtibial, OI: outside-in.

place femoral tunnel most freely and known to have the ability to better restore native anatomy of the $\mathrm{ACL}^{21)}$. Anderson et al. ${ }^{22)}$ reported that the grafts placed with independent drilling had better horizontal alignments similar to the more horizontal and lateral course of the native ACL.

There are some studies reporting femoral tunnel angles measured using simple radiographs. Chang et al. ${ }^{23)}$ reported that the mean coronal plane angle of the femoral tunnel was $61.7^{\circ}$ with the transtibial techniques and $55.9^{\circ}$ with the anteromedial portal technique. Dargel et al. $^{24)}$ reported the angle as $58.8^{\circ}$ and $50.9^{\circ}$, respectively, and Bedi et al. ${ }^{25)}$ reported the values as $54.1^{\circ}$ and $45.9^{\circ}$, respectively. In a study using recently developed transparent 3D CT technique, Takeda et al. ${ }^{14)}$ reported that the mean coronal and sagittal plane femoral tunnel angles were $49^{\circ}$ and $39^{\circ}$, respectively, with the use of the transtibial technique and $32.5^{\circ}$ and $31^{\circ}$, respectively, with the use of the anteromedial portal technique. They also reported the mean coronal and sagittal plane tibial tunnel angles were $57.5^{\circ}$ and $58^{\circ}$, respectively in the knees with the transtibial technique and $67.5^{\circ}$ and $65.5^{\circ}$, respectively in the knees with the anteromedial portal technique. In our study where the outside-in tibial tunnel-independent technique was utilized, the mean femoral tunnel angle was $25.57^{\circ}$ on the AP radiograph and $65.31^{\circ}$ on the lateral radiograph. The mean tibial tunnel angle was $59.16^{\circ}$ on the AP radiograph and $59.05^{\circ}$ on the lateral radiograph. The femoral tunnel showed more horizontal orientation in the coronal plane compared with the transtibial technique, and more horizontal orientation in the sagittal plane compared with the anteromedial portal technique. It was thought to be possible because the independent technique can be most effective for unconstrained orientation of the femoral tunnel during drilling and for more oblique placement of the femoral tunnel in the lateral femoral aspect. However, in our study, these features of the independent technique led to conflicting results with the transtibial technique. In this study, more horizontally oriented femoral tunnels in the coronal plane were significantly associated with the greater femoral tunnel enlargement. This phenomenon was thought to be related to the substantial space in the distal aspect of the femoral tunnel created due to acute bending in graft course, compressive force, and stress concentration to the opposite edge of the tunnel.

There are several limitations to the present study. First, simple radiographs have limitations on detection of tunnel position and orientation due to limited accuracy and high incidence of abnormally rotated projections. Radiographic measurement of tunnel enlargement and tunnel angle cannot be as accurate as magnetic resonance imaging or CT measurement, which we would use in future studies. And the tunnel angle was not shown three-dimensionally for the same reason. However, the two-dimensional data are somewhat useful in intraoperative or postoperative evaluations based on plain radiographs. Second, we used reference data from cadaveric studies instead of the patient's own native ACL footprint for comparison of tunnel placement. Third, we did not consider the anatomical factors such as the shape and size of the femoral condyle that could influence the outcomes. Forth, the relatively small number of patients and short follow-up period might be insufficient for evaluation of outcome, and more longterm follow-up studies including larger populations should be performed in future studies. Finally, we did not correlate the ra- 
diologic changes with clinical outcomes such as functional scores.

\section{Conclusions}

More anterior and higher position of the femoral tunnel and more horizontal orientation of the femoral tunnel in the coronal plane were related to the greater femoral tunnel enlargement in single-bundle ACL reconstruction using the outside-in tibial tunnel-independent technique.

\section{Conflict of Interest}

No potential conflict of interest relevant to this article was reported.

\section{References}

1. Siebold R. Observations on bone tunnel enlargement after double-bundle anterior cruciate ligament reconstruction. Arthroscopy. 2007;23:291-8.

2. Wilson TC, Kantaras A, Atay A, Johnson DL. Tunnel enlargement after anterior cruciate ligament surgery. Am J Sports Med. 2004;32:543-9.

3. Forsythe B, Kopf S, Wong AK, Martins CA, Anderst W, Tashman S, Fu FH. The location of femoral and tibial tunnels in anatomic double-bundle anterior cruciate ligament reconstruction analyzed by three-dimensional computed tomography models. J Bone Joint Surg Am. 2010;92:1418-26.

4. Ahn JH, Jeong HJ, Ko CS, Ko TS, Kim JH. Three-dimensional reconstruction computed tomography evaluation of tunnel location during single-bundle anterior cruciate ligament reconstruction: a comparison of transtibial and 2-incision tibial tunnel-independent techniques. Clin Orthop Surg. 2013;5:26-35.

5. Kopf S, Forsythe B, Wong AK, Tashman S, Anderst W, Irrgang JJ, Fu FH. Nonanatomic tunnel position in traditional transtibial single-bundle anterior cruciate ligament reconstruction evaluated by three-dimensional computed tomography. J Bone Joint Surg Am. 2010;92:1427-31.

6. Arnold MP, Kooloos J, van Kampen A. Single-incision technique misses the anatomical femoral anterior cruciate ligament insertion: a cadaver study. Knee Surg Sports Traumatol Arthrosc. 2001;9:194-9.

7. Tsukada H, Ishibashi Y, Tsuda E, Fukuda A, Toh S. Anatomical analysis of the anterior cruciate ligament femoral and tibial footprints. J Orthop Sci. 2008;13:122-9.
8. Bernard M, Hertel P, Hornung H, Cierpinski T. Femoral insertion of the ACL. Radiographic quadrant method. Am J Knee Surg. 1997;10:14-21.

9. Tsuda E, Ishibashi Y, Fukuda A, Yamamoto Y, Tsukada H, Ono S. Tunnel position and relationship to postoperative knee laxity after double-bundle anterior cruciate ligament reconstruction with a transtibial technique. Am J Sports Med. 2010;38:698-706.

10. Buelow JU, Siebold R, Ellermann A. A prospective evaluation of tunnel enlargement in anterior cruciate ligament reconstruction with hamstrings: extracortical versus anatomical fixation. Knee Surg Sports Traumatol Arthrosc. 2002; 10:80-5.

11. Jagodzinski M, Foerstemann T, Mall G, Krettek C, Bosch U, Paessler HH. Analysis of forces of ACL reconstructions at the tunnel entrance: is tunnel enlargement a biomechanical problem? J Biomech. 2005;38:23-31.

12. Colombet P, Robinson J, Christel P, Franceschi JP, Djian P, Bellier G, Sbihi A. Morphology of anterior cruciate ligament attachments for anatomic reconstruction: a cadaveric dissection and radiographic study. Arthroscopy. 2006;22:984-92.

13. Zantop T, Wellmann M, Fu FH, Petersen W. Tunnel positioning of anteromedial and posterolateral bundles in anatomic anterior cruciate ligament reconstruction: anatomic and radiographic findings. Am J Sports Med. 2008;36:65-72.

14. Takeda Y, Iwame T, Takasago T, Kondo K, Goto T, Fujii $\mathrm{K}$, Naruse A. Comparison of tunnel orientation between transtibial and anteromedial portal techniques for anatomic double-bundle anterior cruciate ligament reconstruction using 3-dimensional computed tomography. Arthroscopy. 2013;29:195-204.

15. Kawaguchi Y, Kondo E, Onodera J, Sasaki T, Yagi T, Yasuda $\mathrm{K}$. Tunnel enlargement and coalition after anatomic doublebundle anterior cruciate ligament reconstruction with hamstring tendon autografts: a computed tomography study. Orthopaedic J Sports Med. 2013;1:1-9.

16. Lee SR, Jang HW, Lee DW, Nam SW, Ha JK, Kim JG. Evaluation of femoral tunnel positioning using 3-dimensional computed tomography and radiographs after single bundle anterior cruciate ligament reconstruction with modified transtibial technique. Clin Orthop Surg. 2013;5:188-94.

17. Youm YS, Cho SD, Eo J, Lee KJ, Jung KH, Cha JR. 3D CT analysis of femoral and tibial tunnel positions after modified transtibial single bundle ACL reconstruction with varus and internal rotation of the tibia. Knee. 2013;20:272-6.

18. Xu Y, Ao Y, Wang J, Yu J, Cui G. Relation of tunnel enlarge- 


\section{Ko et al. The Correlation of Tunnel Position, Orientation and Enlargement in ACLR}

ment and tunnel placement after single-bundle anterior cruciate ligament reconstruction. Arthroscopy. 2011;27:923-32.

19. Chang MJ, Chang CB, Won HH, Je MS, Kim TK. Anteromedial portal versus outside-in technique for creating femoral tunnels in anatomic anterior cruciate ligament reconstructions. Arthroscopy. 2013;29:1533-9.

20. Basdekis G, Abisafi C, Christel P. Influence of knee flexion angle on femoral tunnel characteristics when drilled through the anteromedial portal during anterior cruciate ligament reconstruction. Arthroscopy. 2008;24:459-64.

21. Abebe ES, Moorman CT 3rd, Dziedzic TS, Spritzer CE, Cothran RL, Taylor DC, Garrett WE Jr, DeFrate LE. Femoral tunnel placement during anterior cruciate ligament reconstruction: an in vivo imaging analysis comparing transtibial and 2-incision tibial tunnel-independent techniques. Am J Sports Med. 2009;37:1904-11.

22. Anderson AF, Snyder RB, Lipscomb AB Jr. Anterior cruciate ligament reconstruction: a prospective randomized study of three surgical methods. Am J Sports Med. 2001;29:272-9.

23. Chang CB, Choi JY, Koh IJ, Lee KJ, Lee KH, Kim TK. Comparisons of femoral tunnel position and length in anterior cruciate ligament reconstruction: modified transtibial versus anteromedial portal techniques. Arthroscopy. 2011;27:138994.

24. Dargel J, Schmidt-Wiethoff R, Fischer S, Mader K, Koebke J, Schneider T. Femoral bone tunnel placement using the transtibial tunnel or the anteromedial portal in ACL reconstruction: a radiographic evaluation. Knee Surg Sports Traumatol Arthrosc. 2009;17:220-7.

25. Bedi A, Musahl V, Steuber V, Kendoff D, Choi D, Allen AA, Pearle AD, Altchek DW. Transtibial versus anteromedial portal reaming in anterior cruciate ligament reconstruction: an anatomic and biomechanical evaluation of surgical technique. Arthroscopy. 2011;27:380-90. 